\title{
Editorial
}

\section{Promoting Rational Prescribing Among Medical Practitioners}

\author{
MI Khan ${ }^{1}, \mathrm{~N} \mathrm{Ara}^{2}$
}

Appropriate use of drugs is an essential element in achieving quality of health and medical care for patients and the community as a whole. Inappropriate, ineffective and economically nonviable use of medicines is often observed in health care throughout the world. WHO defined rational use of drug as "Rational use of drugs requires that patients receive medications appropriate to their clinical needs, in doses that meet their own individual requirements for an adequate period of time, and the lowest cost to them and their community." In simplest words, rational use means prescribing right drug, in adequate dose for the sufficient duration and appropriate to the clinical need of the patient at lowest cost. Common types of irrational use of medicines include: use of too many medicines per patient ("poly-pharmacy"); inappropriate use of antimicrobials, often in inadequate dosage, for non-bacterial infections; overuse of injections when oral formulations would be more appropriate; failure to prescribe in accordance with clinical guidelines; inappropriate selfmedication, often of prescription-only medicines; non-adherence to dosing regimes. WHO estimates that more than half of all medicines are prescribed, dispensed or sold inappropriately, and that half of all patients fail to take them correctly and about onethird of the world's population lacks access to essential medicines. ${ }^{2}$ There are many different factors which affect the irrational use of drugs like those deriving from patients, chemists shop, prescribers, the workplace, the supply system, industry influences, regulation, drug information and misinformation. Irrational drug use can have various consequences for the patient, the public, the health system and even in the economy. This can lead to increased morbidity and mortality, wastase of resources, increased risk of adverse drug reactions and the emergence of drug resistance.

1. Dr. Md. Ismail Khan.

Professor, Department of Pharmacology, Dhaka Medical College and Dean, Faculty of Medicine, University of Dhaka

2. Dr. Nazma Ara.

Associate Professor, Dept. of Pharmacology, Delta Medical College, Mirpur, Dhaka.

6
In Bangladesh it is also an emerging problem where there is a wide disparity in the availability of medicines among cities and villages and prescriptions are very much influenced by medical promotion officers of various pharmaceutical companies. In our country medical practitioners are not always aware of number of drugs given per prescription, sometimes improper antibiotics are given to treat infection, given drugs of doubtful efficacy and often failed to prescribe the right drug for a specific condition. Many doctors are not used to prescribe from essential drug lists (EDL) or do not follow Standard Treatment Guidelines (STG). Hundreds of formulations of vitamins, tonics and multi-drug combinations are manufactured and marketed here. There is fierce competition amongst drug companies and they encourage doctors to prescribe branded medicines.

Today rational use of medicine is an issue of the utmost importance. So we should have the responsibility to prescribe rationally. Prescribing is a complex task requiring: diagnostic skills, knowledge of medicines, an understanding of the principles of clinical pharmacology, communication skills, appreciation of risk and uncertainty. The accumulation of clinical trials' data on modern therapies might have been expected to provide sufficient evidence to support most clinical decisions. In fact, clinicians prescribe in varied circumstances, often in the absence of evidence and rational prescribing decisions must be based on knowledge interpreted in the light of many other factors. Rational prescribers should attempt to: 1) maximize clinical effectiveness 2) minimize harms 3) avoid wasting limited healthcare resources 4) respect patient's choice. Rational prescribing normally follows a logical sequence from diagnosis to follow-up.

Irrational prescribing is a habit that is difficult to cure. However prevention is possible. ${ }^{4}$ WHO advocates $12 \mathrm{key}$ interventions to promote more rational use of medicines as follows ${ }^{2}$ -

1. Establiment of a mandated multi-disciplinary national body to coordinate on medicine use policies. 


\section{Use of clinical guidelines}

3. Development and use of national Essential medicines list based on treatments of choice

4. Establishment of drug and therapeutics committees in districts and hospitals

5. Problem-based pharmacotherapy training in undergraduate curricula

6. Continuing in-service medical education as a licensure requirement

\section{Supervision, audit and feedback}

8. Use of independent information on medicines

9. Public education about medicines with major side effects.

\section{Avoidance of perverse financial incentives}

\section{Use of appropriate and enforced regulation}

12. Sufficient government expenditure to ensure availability of medicines and staff

Promoting the rational use of medicines will results in improved quality, increased accessibility and better quality of life for the community. The growing concern is not only for promotion of appropriate use of pharmaceuticals in the health care delivery and its economic considerations but also to provide health related quality of life (HRQL) for a community. There is a need to carefully monitor and censor misleading claims by the pharmaceutical industry. There is some evidence that interventions such as short problem based learning course in pharmacotherapy in medical education and rational use focused workshops can improve prescription behaviour and skills. There is a need to strengthen the mechanism for continuing professional development of practitioners to ensure that they have the necessary knowledge and skills to prescribe rationally. Prescribers should undergo a continuing medical education (CME) course once in a year on newer drugs and different drug combinations, introduced into market.Bangladesh National Formulary ( BDNF) should be distributed to young doctors and general practitioners to provide unbiased drug information and it need to be updated every year. Adverse drug reactions (ADR) reporting should be made mandatory. Pharmacovigilance should be made more effective. Hospitals should constitute Drugs and Therapeutics review committee to rationalize prescribing. Patterns however undergraduate and postgraduate institutes must take the responsibility of training students and young doctors how to assess new drug combinations more logically. Unless we encourage our students to think rationally and independently this menace will continue to grow.

Finally, all healthcare systems have limited resources. The rapidly increasing cost of medicines may force the prescribers to consider cost effectiveness as a factor in drug selection. Devising local formularies, following STG and creating National institute for Health and Clinical Excellence can help doctors to prescribe more rationally. Perhaps the most obvious example of cost effective prescribing is selecting a generic rather than a branded drug from the same therapeutic class. The program of RUM would be initiated and implemented widely at different centers in our country. We have to establish a mandated multidisciplinary national body to regulate and coordinate all activities with sufficient government funding which may be a way to success.

\section{References:}

1.World Health Organization. The Rational Use of Drugs. Report of the Conference of Experts. Geneva. World Health Organization; 1985.

2.Promoting Rational Use of Medicines: Core Components - WHO Policy Perspectives on Medicines,No.05, Document WHO/EDM/2002.3. Geneva, WHO, 2002. Available atURL: http//www.who.int/medicines

3. Rational prescribing: the principles of drug selection. Clinical Medicine. Oct 2009 by Maxwell, Simon

4. Hogerzeil HV. Promoting rational prescribing-an international perspective British Journal of Clinical Pharmacology 1995;39:1-6.

5.Thomas M, Cherian AM, MathaiD. Measuring the impact of focused workshop on rational drug use. Top Doct 1997; 27: 206-10. 